\title{
Heterogeneity Induced Effective Repulsion and Its Effects on Equilibrium Binding Fraction of a Divalent Receptor
}

\author{
Bo Jung Park, Won Jung, and Jaeyoung Sung* \\ Department of Chemistry, Chung-Ang University, Seoul 156-756, Korea. *E-mail: jaeyoung@cau.ac.kr \\ Received October 24, 2009, Accepted December 31, 2009
}

Key Words: Adsorption isotherm, Heterogeneous binding affinity, $\Theta_{0}$ plot, Interaction

Binding of substrate molecules to a limited number of receptors or binding sites is a ubiquitous phenomenon commonly observed across diverse fields of science. When the binding affinity of binding sites to substrate molecules is homogeneous and the interactions between the bound substrate molecules are negligible, the dependence of the equilibrium fraction of the bound sites on the substrate concentration can be described by the classic Langmuir's model. ${ }^{1}$ However, microscopic interactions between substrate molecules in a receptor and heterogeneous affinities of binding sites to substrate molecules make the shape of adsorption isotherm of the receptor significantly distinct from the Langmuir isotherm. ${ }^{2,3}$ Numerous systems of receptors or surfaces were reported to be heterogeneous. The interaction of antibodies with antigens, ${ }^{4 \sim 7}$ the gaseous adsorption onto carbon materials ${ }^{8 \sim 12}$ and metal-organic framework, ${ }^{13 \sim 16}$ and the solute adsorption at heterogeneous interface, ${ }^{17}$ constitute only a very few examples of molecular binding to receptors with a distributed affinity. A variety of different theoretical models have been proposed to provide quantitative descriptions of adsorption isotherms of systems with various binding affinity distributions. $^{18 \sim 20}$

Among the most frequently cited models for substrate-receptor systems with distributed binding affinities are Freundlich model, ${ }^{21,22}$ Sips (Langmuir-Freundlich) model, ${ }^{23}$ and DubininRadushkevich model, ${ }^{24}$ but there are many other models proposed in this field, impossible to mention completely here. ${ }^{2,3}$ Each of these models assumes a particular functional form for the adsorption isotherm or, equivalently, for the distribution of binding affinities of receptors to substrates. However, it is not always easy to isolate the correct model for a given experimental system from the shape of the adsorption isotherm. In some cases a given set of experimental data can be explained by several different models, which introduces ambiguity in the interpretation of the experimental data. ${ }^{25}$ One may be able to choose the best model for given adsorption data. Nevertheless, it is often the case that each of different parts of the experimental data has its own best fitting model, ${ }^{26,27}$ which is somewhat expected because there is no a priori reason that the binding affinity distribution of the experimental system coincides with that assumed in the particular model chosen. Very recently, we proposed a novel, model-independent method of quantitative analysis for a substrate-receptor system with an arbitrary affinity distribution on the basis of an exact relationship between the adsorption isotherm and the affinity distribution of the system, ${ }^{28}$ and introduced a new experimental plot, named $\Theta_{0}$ plot, which is useful for representation and characterization of adsorption isotherm data for substrate-receptor systems with distributed binding affinities.

In the present work, we discuss the effects of microscopic interactions between adsorbate molecules on the qualitative shape of $\Theta_{0}$ plot. For the sake of simplicity we consider a simple system of binding sites, each of which offers two binding sites, A and B, to substrate molecules, $S$. The binding affinity $K_{A}$ of binding site A to the substrate molecules may be different from the binding affinity $K_{B}$ of site $\mathrm{B}$, and the substrate molecule bound to site A interacts with that bound to site B through interaction potential $U$. For the latter model, an exact analytic expression is available for the equilibrium fraction of bound sites: $:^{29}$

$$
\theta_{D}=\frac{2^{-1}\left(K_{A}+K_{B}\right)[S]+K_{A} K_{B} \exp (-\beta U)[S]^{2}}{1+\left(K_{A}+K_{B}\right)[S]+K_{A} K_{B} \exp (-\beta U)[S]^{2}},
$$

where $\theta_{D}$ and $[S]$ denote the equilibrium binding fraction of the divalent receptor and the concentration of substrate molecules, respectively. Equation 1 can be rewritten as

$$
\theta_{D}=2^{-1}\left(\frac{K_{-}[S]}{1+K_{-}[S]}+\frac{K_{+}[S]}{1+K_{+}[S]}\right)
$$

where $K_{ \pm}=\bar{K}[1 \pm \Delta]$ with $\bar{K}$ and $\Delta$ being equal to $\bar{K}=\left(K_{A}+\right.$ $\left.K_{B}\right) / 2$ and

$$
\Delta=\left(1-K_{A} K_{B} e^{-\beta U} / \bar{K}^{2}\right)^{1 / 2}
$$

In the absence of any interaction between the adsorbate molecules, $\Delta$ simplifies to $\Delta_{0}=\left|K_{A}-K_{B}\right| /\left(K_{A}+K_{B}\right)$, and we have $K_{+}=\max \left(K_{A}, K_{B}\right)$ and $K_{-}=\min \left(K_{A}, K_{B}\right)$. In the latter case, $\theta_{D}$ given in eq 2 conforms to the adsorption isotherm $\theta$ of a substrate-receptor system with a binding affinity distribution: $P(K):^{2,3}$

$$
\theta=\int_{0}^{\infty} \frac{K[S]}{1+K[S]} P(K) d K
$$

Here $P(K) d K$ denotes the probability of the binding sites with a phenomenological binding constant being between $K$ and $K+d K$. Note that, in the absence of interactions between substrate molecules, $\theta$ in eq 4 reduces to $\theta_{D}$ given in eq 2 when $P(K)$ is given by the following dichotomous binding affinity distribution: $P(K)=2^{-1}\left[\delta\left(K-K_{A}\right)+\delta\left(K-K_{B}\right)\right]$, where $\delta(x)$ denotes Dirac delta function. 
In the presence of interactions between bound substrate molecules, $\Delta$ given in eq 3 can be written as

$$
\Delta=\Delta_{0}\left[1+\frac{4 K_{A} K_{B}\left(1-e^{-\beta U}\right)}{\left(K_{A}-K_{B}\right)^{2}}\right]^{1 / 2}
$$

so that $\Delta$ and, hence, $K_{ \pm}$assume real values when the interaction between the adsorbed substrates is repulsive, i.e. when $U>0$. In the latter case $\theta_{D}$ in eq 2 can still be described by eq 4 with the following dichotomous binding affinity distribution, $P(K)=$ $2^{-1}\left[\delta\left(K-K_{+}\right)+\delta\left(K-K_{-}\right)\right] \equiv P_{ \pm}(K)$, in which the value of the dichotomous stochastic variable $K$ shifts from $K_{A}$ and $K_{B}$ to $K_{+}$ and $K_{-}$due to the presence of the repulsive interaction between bound substrate molecules. Let us define $\theta_{0}$ by

$$
\theta_{0} \equiv \frac{\bar{K}[S]}{1+\bar{K}[S]}
$$

Since $\bar{K}$ can be estimated from the initial slope of adsorption isotherm, i.e. $\bar{K}=\lim _{[S] \rightarrow 0} \partial \theta([S]) / \partial[S],{ }^{28} \theta_{0}$ can be easily calculated for any value of $[S]$, and one can convert the adsorption isotherm data $\{[S], \theta([S])\}$ into data for $\left\{\theta_{0}, \theta\left(\theta_{0}\right)\right\}$. The plot showing the dependence of $\frac{1-\theta\left(\theta_{0}\right)}{1-\theta_{0}}$ on $\theta_{0}^{2}$ is called $\Theta_{0}$ plot. $^{28}$ From eqs 2 and 6, we obtain

$$
\frac{1-\theta_{D}}{1-\theta_{0}}=\frac{1}{1-\left(\theta_{0} \Delta\right)^{2}}
$$

for the model system with the repulsive interaction potential $U$. Note that $\Delta^{2}$ is the same as the standardized variance $<\delta x^{2}>$ $\left(\equiv\left\langle[(K / \bar{K})-1]^{2}\right\rangle\right)$ of the effective binding affinity distribution $P_{ \pm}(K)$ for the system with repulsive interactions between substrate molecules. As a matter of fact, $\Delta^{2}$ or $<\delta x^{2}>$ is identically equal to the initial slope of the result curve in $\Theta_{0}$ plot, i.e.

$$
<\delta x^{2}>=\lim _{\theta_{0} \rightarrow 0} \frac{\partial}{\partial\left(\theta_{0}^{2}\right)} \frac{1-\theta\left(\theta_{0}\right)}{1-\theta_{0}} .
$$

It can be shown that eq 8 holds for an arbitrary binding affinity distribution, $P(K) .{ }^{28}$ In the presence of repulsive interactions $(U>0)$ between bound substrate molecules, the value of $\Delta^{2}$ given in eq 5 increases with $\beta U$ from $\Delta_{0}^{2}$ to 1 , which is always positive.

In comparison, when the attractive interactions $(U<0)$ between the adsorbed substrate molecules are strong enough to satisfy the following condition: $\exp (-\beta U)>\bar{K}^{2} /\left(K_{A} K_{B}\right), \Delta$ given in eq 3 has a pure imaginary value so that $K_{ \pm}$becomes a complex quantity. In this case, one can no longer represent $\theta_{D}$ in eq 2 by eq 4 with $P(K)=P_{ \pm}(K)$, the line integral along the real axis. Nevertheless, eq 7 holds still for the system under the strong attractive interaction potential for which $\Delta$ is pure imaginary, i.e. $\Delta=i|\Delta|$. For the latter case eq 7 reads as

$$
\frac{1-\theta_{D}}{1-\theta_{0}}=\frac{1}{1+\left(\theta_{0}|\Delta|\right)^{2}} \quad\left[\exp (-\beta U) \geq \bar{K}^{2} /\left(K_{A} K_{B}\right)\right]
$$

Equation 9 tells us that the curves in $\Theta_{0}$ plot for the systems with such strong attractive interactions between adsorbate molecules decrease with $\theta_{0}^{2}$ in contrast to those for the systems with repulsive interactions between bound substrate molecules or with distributed binding affinities.

From eq 3 one can show that the initial slope $\Delta^{2}$ of the $\Theta_{0}$ plot for our model can be written as $1-\exp \left\{-\beta\left[U+U_{h}(\beta)\right]\right\}$ with $U_{h}$ being the effective potential defined by

$$
U_{h} \equiv 2 \beta^{-1} \ln [\operatorname{Cosh}(h / 2)]
$$

In eq $10 h$ is given by $h=\operatorname{Ln}\left(K_{B} / K_{A}\right)$, which is the same as the difference of the binding free energy $g_{A}$ of site A from the binding free energy $g_{B}$ of site B in unit of thermal energy, i.e. $h=\beta\left(g_{A}-g_{B}\right)$. The effective potential $U_{h}$ given in eq 10 takes into account the effects of heterogeneity between the binding affinity of site $\mathrm{A}$ and that of site $\mathrm{B}$ on the initial slope $\Delta^{2}$ in $\Theta_{0}$ plot.

We show the dependence of $U_{h}$ on $h$ in Figure 1(a) and the dependence of the initial slope $\Delta^{2}$ in the $\Theta_{0}$ plot on $h$ and $U$ in Figure 1(b). As shown $U_{h}$ is an even function of $h$ that increases with the magnitude of $h$, and it vanishes for the homogeneous case with $K_{A}$ being equal to $K_{B}$ or with $h$ being equal to 0 . Note that $U_{h}$ always has a non-negative value. In small $h$ regime $U_{h}$ can be approximated as the quadratic function of $h, U_{h} \cong \beta^{-1}(h / 2)^{2}$; in comparison, in large $h$ regime, it can be approximated as a linear function of $h, U_{h} \cong h / \beta$. As shown in Figure 1(b), the initial slope $\Delta^{2}$ of the $\Theta_{0}$ plot for our model is positive if the microscopic interaction potential $U$ is greater than $-U_{h}(\beta)$. Since $U_{h}(\beta)$ has a non-negative value in any case, the latter condition is satisfied for any repulsive interaction potential $U$ with the positive sign. In comparison, for the system with an attractive interaction potential $(U<0)$, the initial slope $\Delta^{2}$ in the $\Theta_{0}$ plot has a negative value when $U<$ $-U_{h}(\beta)$, has a positive value when $U>-U_{h}(\beta)$, and vanishes when $U=-U_{h}(\beta)$, as shown in Figure 1(b). Therefore, the negative initial slope in the $\Theta_{0}$ plot is the sufficient condition for the presence of the attractive interactions between bound substrate molecules.

Although eq 9 is obtained for a very simple model, it provides the correct qualitative description for a general system in the low substrate concentration regime or in the small $\theta_{0}$ regime, in which interactions among more than two substrate molecules are negligible. Therefore, the data curves in the $\Theta_{0}$ plot have the negative initial slope for any system with strong attractive interactions between adsorbed substrate molecules. As an example, in Figure 2, we show the $\Theta_{0}$ plot constructed from the adsorption isotherm of $\mathrm{CO}_{2}$ molecules onto the metal organic framework called IRMOF-1 reported in Figure 1 of ref 15 , in which the authors showed that attractive electrostatic interactions between $\mathrm{CO}_{2}$ molecules plays an important role on the shape of the adsorption isotherm. For the latter system, the values of $\bar{K}$ and the initial slope in the $\Theta_{0}$ plot are 0.00289 bar $^{-1}$ and -6.052 , respectively. As the experimental system is much more complex than our model, the data curve in the $\Theta_{0}$ plot of the system is not in quantitative agreement with the prediction of eq 9; however, both shares the same key qualitative 
(a)

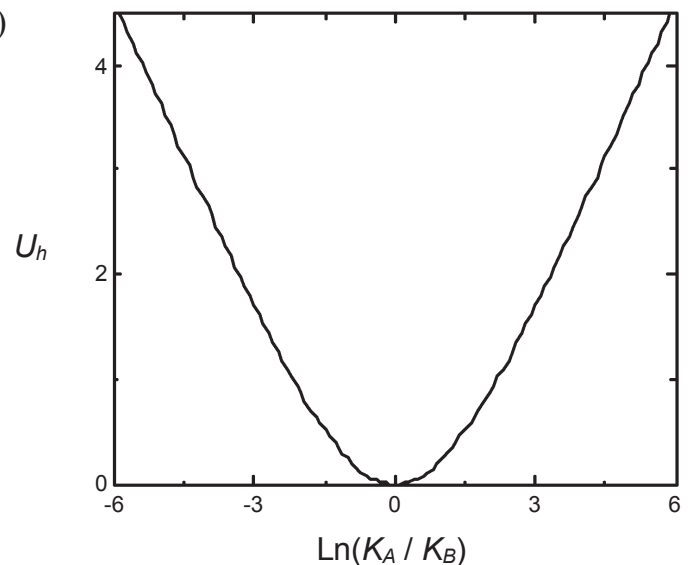

(b)

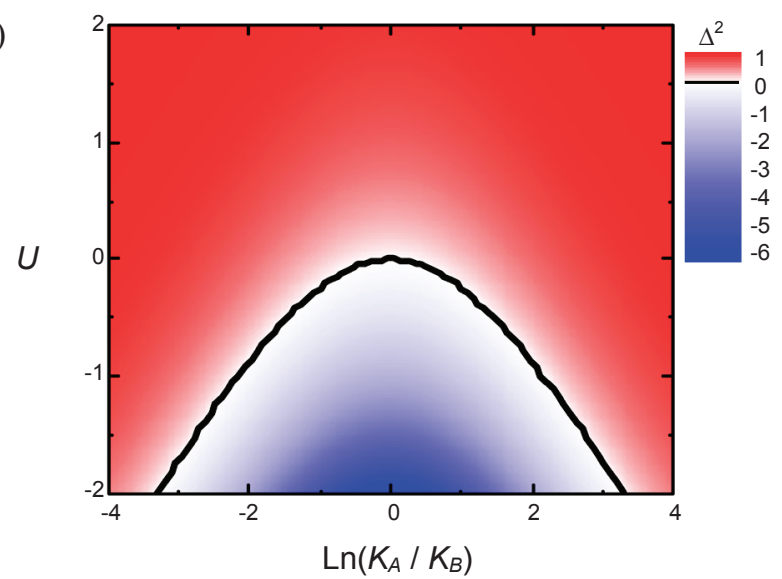

Figure 1. (a) Effective repulsive potential $U_{h}$ due to heterogeneity in the binding affinity for a receptor with two binding sites A and B, given in eq 10. $K_{A(B)}$ is the equilibrium constant for binding of site A(B) in the receptor to substrate molecules. $U_{h}$ increases with the magnitude of $h\left[\equiv \operatorname{Ln}\left(K_{A} / K_{B}\right)\right]$. (b) Dependence of the initial slope $\Delta^{2}$ in the $\Theta_{0}$ plot on the microscopic interaction potential $U$ between the substrate molecules bound to site $\mathrm{A}$ and that bound to site $\mathrm{B}$. The sign of $\Delta^{2}$ is positive when $U>-U_{h}$ but negative when $U<-U_{h} . \Delta^{2}$ vanishes when $U=U_{h}$. The unit of $U_{h}$ and $U$ is thermal energy, $k_{B} T$.

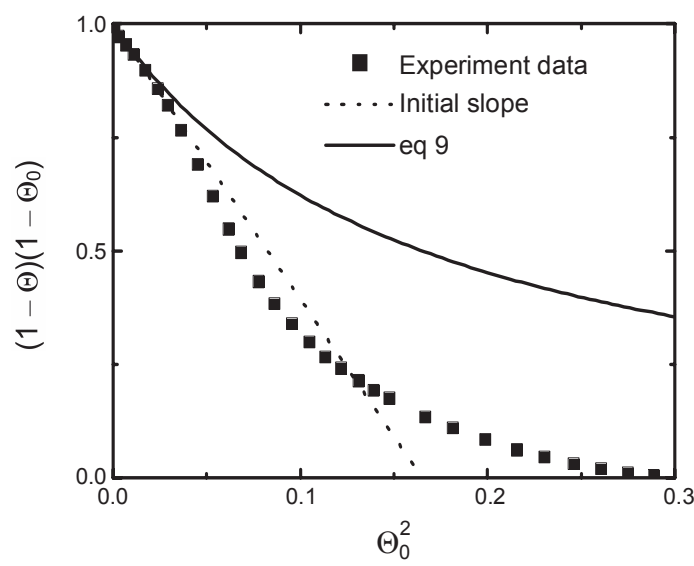

Figure 2. The $\Theta_{0}$ plot for the adsorption of carbon dioxide molecules onto metal-organic framework IRMOF-1 reported in Figure 1 (Table S7) of ref 14. The initial slope of the data in the $\Theta_{0}$ plot is negative in qualitative agreement with eq 9 for our simple model. feature, the negative initial slope in the $\Theta_{0}$ plot due to the attractive interactions between adsorbate molecules.

We have discussed the effects of microscopic interactions on the shape of $\Theta_{0}$ plot for the simple receptor model with two heterogeneous binding sites. It is straightforward to generalize the present discussion for the more complex interaction models in which an arbitrary number of bound substrate molecules interact with an arbitrary microscopic potential. Research in this direction is currently under going on.

Acknowledgments. This work is supported by Chung-Ang University Research Grant in 2008.

\section{Reference}

1. Langmuir, I. J. Am. Chem. Soc. 1917, 39, 1848.

2. Jaroniec, M.; Madey, R. Physical Adsorption on Heterogeneous Solids; Elsevier: Amsterdam-Oxford-New York-Tokyo, 1988.

3. Toth, J. Adsorption: theory, modeling, and analysis, Surfactant Science Series; Marcel Dekker: New York-Basel, 2002; Vol. 107.

4. Pauling, L.; Pressman, D.; Grossberg, A. L. J. Am. Chem. Soc. 1944, 66, 784 .

5. Karush, F.; Sonenberg, M. J. Am. Chem. Soc. 1949, 71, 1369.

6. Karush, F. J. Am. Chem. Soc. 1956, 78, 5519.

7. Temirov, J. P.; Bradbury A. R. M.; Werner J. H. Anal. Chem. 2008, 80,8642 .

8. Wang, H.; Gao, Q.; Hu, J. J. Am. Chem. Soc. 2009, 131, 7016.

9. Yoon, S. B.; Chai, G. S.; Kang, S. K.; Yu, J.-S.; Gierszal, P. G.; Jaroniec, M. J. Am. Chem. Soc. 2005, 127, 4188.

10. Gogotsi, Y.; Dash, R. K.; Yushin, G.; Yildirim, T.; Laudisio, G.; Fischer, J. E. J. Am. Chem. Soc. 2005, 127, 16006.

11. (a) Anson, A.; Callejas, M. A.; Benito, A. M.; Maser, W. K.; Izquierdo, M. T.; Rubio, B.; Jagiello, J.; Thommes, M.; Parra, J. B.; Martínez, M. T. Carbon 2004, 42, 1243-1248. (b) Kajiura, H.; Tsutsui, S.; Kadono, K.; Kakuta, M.; Ata, M.; Murakami, Y. Appl. Phys. Lett. 2003, 82, 1105-1107.

12. Yang, Z.; Xia, Y.; Mokaya, R. J. Am. Chem. Soc. 2007, 129, 1673.

13. Millward, A. R.; Yaghi, O. M. J. Am. Chem. Soc. 2005, 127, 17998.

14. Walton, K. S.; Millward, A. R.; Dubbeldam, D.; Frost, H.; Low, J. J.; Yaghi, O. M.; Snurr. R. Q. J. Am. Chem. Soc. 2008, 130, 406.

15. Caskey, S. R.; Wong-Foy, A. G.; Matzger, A. J. J. Am. Chem. Soc. 2008, 130, 10870 .

16. Ok, K. M.; Sung, J.; Hu, G.; Jacobs, R. M.; O’Hare, D. J. Am. Chem. Soc. 2008, 130, 3762

17. Rudzinski, W.; Charmas, R.; Partyka, S.; Bottero, J. Y. Langmuir 1993, 9, 2641.

18. Joshi, U. D.; Joshi, P. N.; Tamhankar, S. S.; Joshi, V. V.; Shiralkar, V. P. J. Phys. Chem. 2001, 105, 10637.

19. Peterson, A. W.; Wolf, L. K.; Georgiadis. R. M. J. Am. Chem. Soc. 2002, 124, 14601 .

20. Garcés, J. L.; Mas, F. J. Chem. Phys. 2004, 120, 9266.

21. Freundlich, H. Z. Phys. Chem. 1906, 57, 385.

22. Zeldowitsch, J. Acta Physicochim. URSS 1934, 961, 1.

23. Sips, R. J. Chem. Phys. 1948, 16, 490.; ibid 1950, 18, 1024.

24. Dubinin, M. M. In Chemistry and physics of Carbon; Walker, P. L., Jr., Ed., Marcell Dekker: New York, 1966; Vol. 2, p 51.

25. Noroozifar, M.; Khorasani-Motlaph, M.; Fard, P. A. J. of Hazard. Mater. 2009, 166, 1060.

26. Cerofolini, G. F. J. Colloid Interface Sci. 1982, 86, 204.

27. Cerofolini, G. F. In Specialist Periodical Reports: Colloid Sci; Everett, D. H., Ed., The Chemical Society: London, 1982; Vol. 4, pp 59-83.

28. Jung, W.; Park, B. J.; Yang, S.; Jang, S.-K.; Sung, J. Langmuir (Submitted for publication).

29. Bae, J. H.; Lim, Y. R.; Sung, J. Langmuir 2008, 24, 2569. 